\title{
COLLISION STRENGTHS FOR EXCITATION OF \\ FORBIDDEN LINES
}

\author{
S.J. Czyzak and T.K. Krueger and P. de A.P. Martins, H.E. Saraph, \\ M.J. SeAton and J. Shemming* \\ (Dept. of Physics, \\ (Ohio State University, and \\ Aerospace Research Laboratory, \\ Wright-Patterson Air Force Base, U.S.A.) \\ University College London, England)
}

New calculations of collision strengths are being made for ions in configurations $n p^{q}, n=2$ and 3 and $q=1-5$. Wave functions for the entire system are represented by sums over anti-symmetrized products of ion functions and orbitals for the colliding

Table 1

Calculated collision strengths for $k_{3}^{2}=0$

\begin{tabular}{|c|c|c|c|}
\hline Ion & $\Omega(1,2)$ & $\Omega(1,3)$ & $\Omega(2,3)$ \\
\hline $\mathbf{N}^{+}$ & $3 \cdot 14$ & 0.342 & 0.376 \\
\hline $\mathrm{O}^{+2}$ & $2 \cdot 39$ & 0.335 & 0.310 \\
\hline $\mathrm{F}^{+3}$ & 1.93 & $0 \cdot 279$ & 0.237 \\
\hline $\mathrm{Ne}^{+4}$ & $1 \cdot 38$ & $0 \cdot 218$ & $0 \cdot 185$ \\
\hline $\mathrm{O}^{+}$ & 1.43 & 0.428 & 1.70 \\
\hline $\mathrm{F}^{+2}$ & 1.25 & 0.461 & 1.67 \\
\hline $\mathrm{Ne}^{+3}$ & 1.04 & 0.427 & 1.42 \\
\hline $\mathrm{Na}^{+4}$ & 0.836 & 0.359 & 1.22 \\
\hline $\mathrm{F}^{+}$ & $1 \cdot 34$ & $0 \cdot 147$ & $0 \cdot 193$ \\
\hline $\mathrm{Ne}^{+2}$ & 1.27 & $0 \cdot 164$ & $0 \cdot 188$ \\
\hline $\mathrm{Na}^{+3}$ & $1 \cdot 14$ & $0 \cdot 163$ & $0 \cdot 157$ \\
\hline $\mathrm{Mg}^{+4}$ & 0.973 & $0 \cdot 146$ & 0.129 \\
\hline $\mathbf{P}^{+}$ & $6 \cdot 31$ & $1 \cdot 12$ & $1 \cdot 11$ \\
\hline $\mathrm{S}^{+2}$ & 4.97 & 1.07 & 0.961 \\
\hline $\mathrm{Cl}^{+3}$ & 1.99 & 0.328 & 1.03 \\
\hline $\mathrm{Ar}^{+4}$ & $1 \cdot 19$ & 0.141 & 0.945 \\
\hline $\mathrm{S}^{+}$ & 3.07 & 1.28 & $6 \cdot 22$ \\
\hline $\mathrm{Cl}^{+2}$ & $3 \cdot 19$ & 1.97 & $6 \cdot 64$ \\
\hline $\mathrm{Ar}^{+3}$ & 1.43 & 0.645 & 4.92 \\
\hline $\mathbf{K}^{+4}$ & 0.751 & 0.256 & $4 \cdot 24$ \\
\hline $\mathrm{Cl}^{+}$ & 3.94 & 0.412 & 0.749 \\
\hline $\mathrm{Ar}^{+2}$ & $4 \cdot 75$ & 0.724 & 0.665 \\
\hline $\mathrm{K}^{+3}$ & 1.92 & 0.296 & 0.681 \\
\hline $\mathrm{Ca}^{+4}$ & 0.908 & 0.115 & 0.777 \\
\hline
\end{tabular}

* Now at Norwich City College.

Osterbrock and O'Dell (eds.), Planetary Nebulae, 138-142. I.A.U. 
Table 2

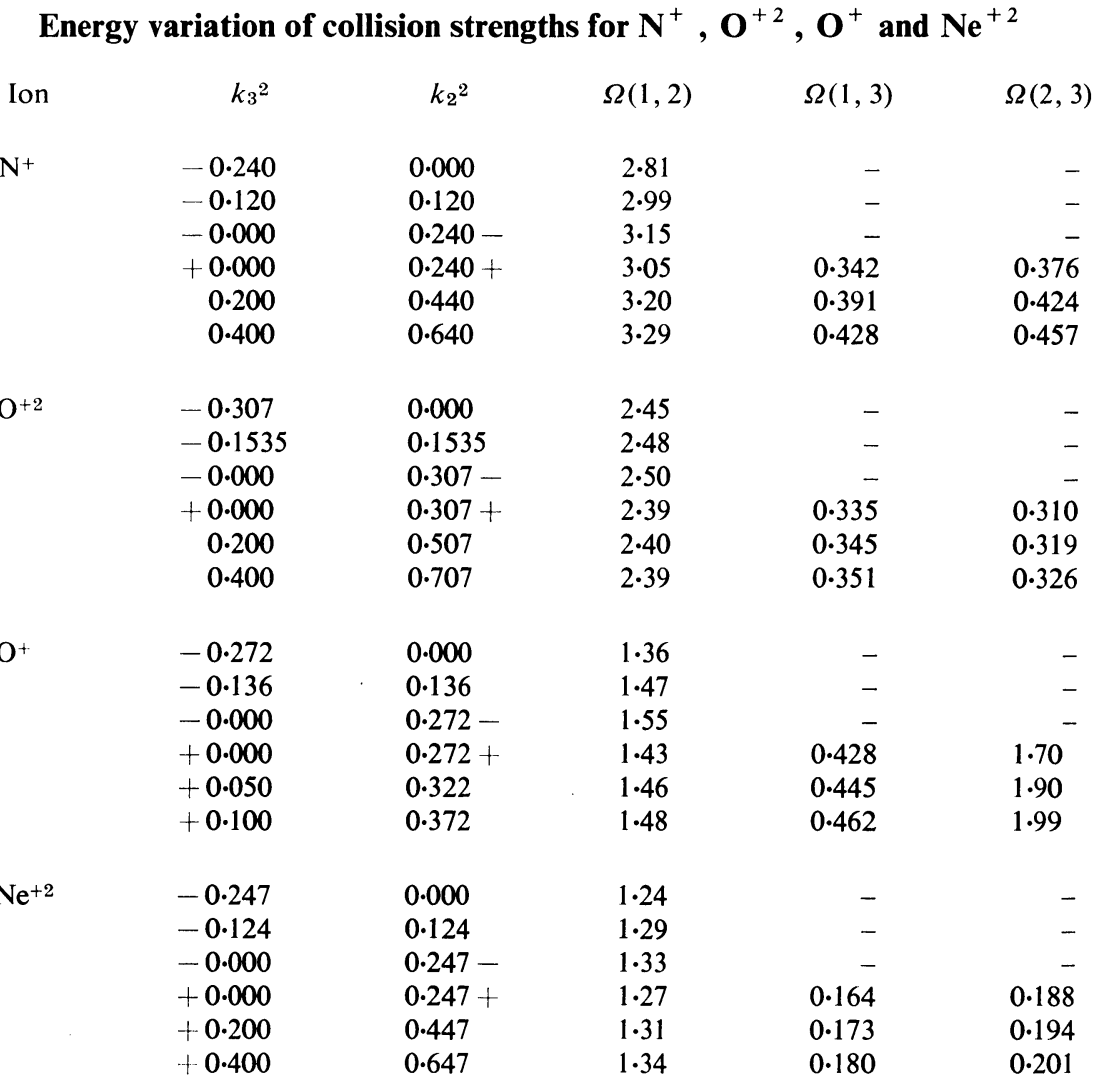

Table 3

Transitions between $\mathrm{O}^{+}$fine-structure states

$i$

$\begin{array}{ll}{ }^{4} S_{3 / 2} & { }^{2} D_{3 / 2} \\ { }^{4} S_{3 / 2} & { }^{2} D_{5 / 2} \\ { }^{4} S_{3 / 2} & { }^{2} P_{1 / 2} \\ { }^{4} S_{3 / 2} & { }^{2} \mathbf{P}_{3 / 2} \\ { }^{2} D_{3 / 2} & { }^{2} D_{5 / 2} \\ { }^{2} D_{3 / 2} & { }^{2} \mathbf{P}_{1 / 2} \\ { }^{2} \mathbf{D}_{3 / 2} & { }^{2} \mathbf{P}_{3 / 2} \\ { }^{2} \mathbf{D}_{5 / 2} & { }^{2} \mathbf{P}_{1 / 2} \\ { }^{2} \mathbf{D}_{5 / 2} & { }^{2} \mathbf{P}_{3 / 2} \\ { }^{2} \mathbf{P}_{1 / 2} & { }^{2} \mathbf{P}_{3 / 2}\end{array}$

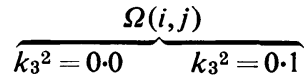

$\begin{array}{ll}0.568 & 0.589 \\ 0.852 & 0.884 \\ 0.144 & 0.156 \\ 0.288 & 0.311 \\ 0.890 & 0.924 \\ 0.353 & 0.378 \\ 0.481 & 0.543 \\ 0.342 & 0.398 \\ 0.909 & 0.999 \\ 0.257 & 0.283\end{array}$


Table 4

\begin{tabular}{lcccc}
\multicolumn{5}{c}{ Transitions within ${ }^{3} \mathbf{P}$ terms } \\
Ion & $k_{3}{ }^{2}$ & $\Omega\left({ }^{3} \mathrm{P}_{0},{ }^{3} \mathrm{P}_{1}\right)$ & $\Omega\left({ }^{3} \mathrm{P}_{0},{ }^{3} \mathrm{P}_{2}\right)$ & $\Omega\left({ }^{3} \mathrm{P}_{1},{ }^{3} \mathrm{P}_{2}\right)$ \\
$\mathrm{N}^{+}$ & 0.0 & .401 & .279 & $1 \cdot 128$ \\
& $0 \cdot 1$ & .424 & .282 & $1 \cdot 164$ \\
$\mathrm{O}^{+2}$ & 0.0 & .376 & .213 & .948 \\
& 0.4 & .391 & .207 & .954 \\
$\mathrm{Ne}^{+2}$ & 0.0 & .185 & .131 & .527 \\
& 0.4 & .200 & .134 & .551
\end{tabular}

electron, and sets of coupled integro-differential equations are obtained for the colliding electron radial functions. The main approximation is neglect of coupling to configurations other than $n p^{q}$. Calculations are made using improved forms of the exact resonance and distorted wave approximations. For the $2 p^{q}$ ions it is found that the dominant contributions come from the $p$-waves; the exact resonance approximation is used for the $p$-waves and the distorted wave approximation is used for all other partial waves. It is considered that the results obtained should approximate closely to those which would be obtained from full solutions of the coupled equations. For the $3 p^{q}$ ions the dominant contributions come from the $d$-waves; for these ions the distorted wave approximation is used for all partial waves.

We give some results which have been obtained to date for $q=2,3$ and 4 . These configurations have three terms $\left({ }^{3} \mathrm{P},{ }^{1} \mathrm{D}\right.$ and ${ }^{1} \mathrm{~S}$ for $q=2$ and $4 ;{ }^{4} \mathrm{~S},{ }^{2} \mathrm{D}$ and ${ }^{2} \mathrm{P}$ for $q=3$ ) denoted, in order of increasing excitation energy, by $i=1,2$ and 3. Energies of the colliding electron, in Rydbergs, are denoted by $k_{i}^{2}$. Table 1 gives the calculated collision strengths for $k_{3}^{2}=0$. The collision strength $\Omega(1,2)$ has a complicated resonance structure for $k_{3}^{2}<0$; we average over the resonances using a formula of Gailitis (1963). Table 2 gives collision strengths for $\mathrm{N}^{+}, \mathrm{O}^{+2}, \mathrm{O}^{+}$and $\mathrm{Ne}^{+2}$ as functions of energy. Table 3 gives results for transitions between the fine structure states of $\mathrm{O}^{+}$, which are required for the interpretation of [OII] $\lambda 3726 / \lambda 3729$ intensity ratios; further calculations of $\Omega\left({ }^{2} \mathrm{D}_{3 / 2},{ }^{2} \mathrm{D}_{5 / 2}\right)$ are being made for energies $k_{3}^{2}<0$. Table 4 gives results for transitions between the fine structure states in the ${ }^{3} \mathrm{P}$ terms of $\mathrm{N}^{+}, \mathrm{O}^{+2}$ and $\mathrm{Ne}^{+2}$.

The reactance matrices calculated for collisions with an ion $X^{+m}$ can be extrapolated to negative energies and used to calculate the positions of bound states of $X^{+(m-1)}$. A comparison with observed positions gives a guide to the accuracy of the calculations. As a final step, parameters may be introduced into the expressions for the reactance matrices and adjusted so as to obtain an improved agreement with observed bound states, and the adjusted matrices may be used to obtain improved collision strengths. This method has, so far, been applied successfully only to the $p$-wave contributions for the $2 p^{q}$ ions. The calculations of bound-state energy levels are made in intermediate coupling and it is found that the differences between observed and calculated energies are practically the same for all levels within any one SL term. Table 5 
Table 5

Effective quantum numbers $v$ for $2 p^{2}$ np SL states of $O^{+}$referred to the $O^{+2} 2 p^{2}{ }^{3} P$ series limit

$\begin{array}{llcr}n & \mathrm{SL} & v_{\text {calc }}-v_{\text {ohs. }} & v_{\mathrm{adj} .}-v_{\text {obs. }} \\ 3 & { }^{2} \mathrm{~S} & .0432 & .0078 \\ 3 & { }^{2} \mathrm{P} & .0323 & -.0077 \\ 3^{\prime} & { }^{2} \mathrm{P} & .0632 & -.0090 \\ 4 & { }^{2} \mathrm{P} & .0361 & -.0027 \\ 3 & { }^{2} \mathrm{D} & .0328 & .0017 \\ 3^{\prime} & { }^{2} \mathrm{D} & .0728 & .0043 \\ 4 & { }^{2} \mathrm{D} & .0365 & .0035 \\ 5 & { }^{2} \mathrm{D} & .0327 & .0005 \\ 3^{\prime} & { }^{2} \mathrm{~F} & .0410 & -.0170 \\ 3 & { }^{4} \mathrm{~S} & .0652 & .0270 \\ 3 & { }^{4} \mathrm{P} & .0280 & -.0070 \\ 5 & { }^{4} \mathrm{P} & .0253 & -.0047 \\ 3 & { }^{4} \mathrm{D} & .0299 & -.0054 \\ 4 & { }^{4} \mathrm{D} & .0278 & -.0037 \\ 5 & { }^{4} \mathrm{D} & .0269 & -.0033\end{array}$

gives the differences between calculated and observed effective quantum numbers for $152 p^{2} n p \mathrm{SL}$ terms in $\mathrm{O}^{+}$, and differences obtained when 4 parameters are introduced into the expressions for the reactance matrices and adjusted so as to give a least squares fit in the energy levels. Table 6 gives some results for the calculated and adjust-

Table 6

$p$-Wave contributions to collision strengths, $k_{3}{ }^{2}=0$

\begin{tabular}{lllll} 
& \multicolumn{2}{c}{$\Omega^{\mathrm{pp}}(1,3)$} \\
\cline { 2 - 3 } & Calc. & Adj. & Calc. & Adj. \\
$\mathrm{N}^{+}$ & 2.657 & 2.678 & 0.288 & 0.480 \\
$\mathrm{O}^{+2}$ & 1.658 & 1.582 & 0.204 & 0.195 \\
$\mathrm{~F}^{+3}$ & 1.045 & 0.802 & 0.131 & 0.098 \\
& & & & 0.423 \\
$\mathrm{O}^{+}$ & 1.302 & 1.449 & 0.381 & \\
$\mathrm{Ne}^{+2}$ & 0.979 & 1.001 & 0.116 & 0.157 \\
$\mathrm{Na}^{+3}$ & 0.730 & 0.543 & 0.088 & 0.069
\end{tabular}

ed $p$-wave contributions to the collision strengths of various ions. It should be noted that, for the energy differences between the $n p^{q}$ terms, we use calculated values for Tables 1 and 2 and experimental values for Tables 3, 4, 5, and 6 .

Table 7 gives a summary of results obtained in different calculations for $\mathrm{O}^{+2}$ (Seaton, 1953, 1955, 1958; Saraph et al., 1966). It is seen that there is little change in the ratio $\Omega(1,2) / \Omega(1,3)$, which is of importance for the determination of electron tem- 


\section{Table 7}

\section{Comparison of different calculations for $\mathrm{O}^{+2}, \mathrm{k}_{3}{ }^{2}=0$}

$\begin{array}{lcccc} & \Omega(1,2) & \Omega(1,3) & \Omega(2,3) & \Omega(1,2) / \Omega(1,3) \\ \text { Calculated, 1953 } & 1.73 & 0.195 & - & 8 \cdot 87 \\ \text { Calculated, 1955 } & - & - & 0.61 & - \\ \text { Recommended, 1958 } & 1.59 & 0.22 & 0.64 & 7 \cdot 23 \\ \text { Calculated, 1966 } & 2 \cdot 39 & 0.335 & 0.310 & 7 \cdot 14 \\ \text { Adjusted, 1967 } & 2.32 & 0.326 & 0.309 & 7 \cdot 12\end{array}$

peratures. The present results for $\Omega(2,3)$ differ from the earlier results due to the inclusion of exchange for the higher order partial waves.

Some further details on the formulation employed in this work are given by Saraph et al. (1966), together with results for $2 \mathrm{p}^{2}$ ions. Results for $3 \mathrm{p}^{3}$ ions have been given by Czyzak and Krueger (1967) and results for $2 p^{3}, 2 p^{4}, 3 p^{2}$ and $3 p^{4}$ have been reported by Czyzak et al. (1967). Our programme of calculations is being continued and a full account of the work is being prepared for publication.

\section{References}

Czyzak, S.J., Krueger, T. K. (1967) Proc. Phys. Soc., London, 90, 623.

Czyzak, S.J., Krueger, T.K., Saraph, H.E., Shemming, J. (1967) Proc. Phys. Soc., London, 92, 1146.

Gailitis, M. (1963) Soviet Phys. JETP, 17, 1328.

Saraph, H.E., Seaton, M.J., Shemming, J. (1966) Proc. Phys. Soc., 89, 27.

Seaton, M.J. (1953) Proc. R. Soc. London, A218, 400.

Seaton, M.J. (1955) ibid., 231, 37.

Seaton, M.J. (1958) Rev. mod. Phys., 30, 979.

\section{DISCUSSION}

Garstang: The variation of the collision strength as a function of $Z$ along an isoelectronic sequence is of interest. Usually this variation is smooth. Have you found any cases where this variation is not smooth, e.g. by means of irregularities of quantum defects, or any cases where the square roots of the collision strengths pass through a zero?

Seaton: One considers $Z^{2} \Omega$, where $Z$ is the charge; this remains finite as $Z \rightarrow \infty$. More or less irregular variations in $Z^{2} \Omega$ can occur along an isoelectronic sequence. 